\title{
The human Tap protein is a nuclear mRNA export factor that contains novel RNA-binding and nucleocytoplasmic transport sequences
}

\author{
Yibin Kang $^{1}$ and Bryan R. Cullen ${ }^{1-3}$ \\ ${ }^{1}$ Department of Genetics and ${ }^{2}$ Howard Hughes Medical Institute, Duke University Medical Center, \\ Durham, North Carolina 27710 USA
}

\begin{abstract}
The constitutive transport element (CTE) encoded by simian type D retroviruses directs unspliced viral RNAs into a nuclear export pathway that is congruent with the pathway used by cellular mRNAs. Here, we show that quail cells are refractory to CTE function but become highly permissive upon expression of the human Tap protein, a candidate CTE cofactor. Tap contains a novel sequence-specific RNA binding domain that is sufficient for CTE binding but inadequate to support CTE function. Using microinjection assays, we have defined two NLSs and one NES in Tap. Mutational inactivation of the Tap NES, which lies outside the RNA-binding domain, not only blocks Tap function but also generates dominant-negative forms of Tap. Whereas replacement of the Tap NES with the well-defined Rev NES rescues the ability of Tap to support CTE function, this substitution also confers sensitivity to agents that block the activity of Crm1, the Rev NES cofactor. Together, these data validate Tap as the first human sequence-specific nuclear mRNA export factor and identify a novel type of NES that can support nuclear mRNA export but does not act via Crm1.
\end{abstract}

[Key Words: mRNA; nuclear export; nuclear import; retroviral; constitutive transport element]

Received December 30, 1998; revised version accepted March 18, 1999.

Retroviral replication requires the nuclear export and translation of unspliced as well as spliced forms of the initial, genome-length viral RNA transcript. However, cells have developed mechanisms to retain incompletely spliced mRNAs, i.e., mRNAs that contain functional splice sites, in the nucleus to avoid the cytoplasmic translation of cellular pre-mRNAs that would be likely to encode useless, or even deleterious, protein products (Chang and Sharp 1989; Legrain and Rosbash 1989). The ability to access alternative nuclear RNA export pathways that permit the delivery of incompletely spliced viral transcripts to the cytoplasm in the face of this cellular retention mechanism is therefore a general characteristic of retroviruses (for review, see Hammarskjöld 1997; Cullen 1998). This property has made these viruses a useful model system for the identification and study of nuclear RNA export pathways.

The best understood retroviral nuclear RNA export pathway is the one used by complex retroviruses such as human immunodeficiency virus type 1 (HIV-1). HIV-1 encodes a regulatory protein, termed Rev, that binds directly to the Rev response element (RRE) RNA target sequence found on all incompletely spliced HIV-1 mRNAs (Malim et al. 1989, 1990). Rev also contains a potent nuclear export signal (NES) (Fischer et al. 1995;

${ }^{3}$ Corresponding author.

E-MAIL culle002@mc.duke.edu; FAX (919) 681-8979.
Wen et al. 1995) that functions as the binding site for a heterodimer consisting of the cellular nuclear export factor Crm1, linked to the GTP-bound form of the Ran nucleocytoplasmic transport cofactor (Fornerod et al. 1997a; Neville et al. 1997; Stade et al. 1997). Crm1 has been proposed to target the resultant ribonucleoprotein complex to the nuclear pore, and hence to the cytoplasm, by binding directly to selected nucleoporins, including Nup214/Can (Fornerod et al. 1997b; Neville et al. 1997).

Whereas complex retroviruses such as HIV-1 encode their own Rev-like nuclear RNA export factor, simple retroviruses encode only the viral structural proteins (Hammarskjöld 1997; Cullen 1998). It was therefore initially unclear how these less complex viruses would be able to achieve the nuclear export of genome-length, unspliced viral RNAs. For at least some simple retroviruses, including particularly type $\mathrm{D}$ simian retroviruses such as Mason-Pfizer monkey virus (MPMV), this question was resolved with the identification of a cis-acting RNA structure, termed the constitutive transport element (CTE), that proved able to induce the efficient nuclear export of unspliced MPMV RNAs (see Fig. 2, below) (Bray et al. 1994). As this export can occur in the absence of viral proteins, CTE function must be mediated by a cellular nuclear export factor.

Because the CTE can substitute for the Rev:RRE interaction in mediating unspliced HIV-1 RNA expression (Bray et al. 1994; Zolotukhin et al. 1994), it seemed pos- 
sible that CTE function might also be dependent on Crm1 function. Several lines of evidence now argue that this is not the case: (1) The drug leptomycin B, which binds to and inactivates Crm1, inhibits Rev but not CTE function (Otero et al. 1998). (2) Similarly, dominantnegative mutants of nucleoporins, such as Nup214/Can, that retain the ability to bind Crm 1 , but are not incorporated into nuclear pores, can specifically block Rev, but not CTE, function (Bogerd et al 1998; Zolotukhin and Felber 1999) . (3) In Xenopus oocytes, high levels of the Rev NES inhibit Rev function but not CTE function, whereas high levels of the CTE inhibit CTE but not Rev function (Pasquinelli et al. 1997; Saavedra et al. 1997). Interestingly, other squelching studies in oocytes have revealed that high levels of the Rev NES can inhibit 5S rRNA and U snRNA export selectively but not cellular mRNA export (Fischer et al. 1995), an observation that is consistent with the lack of effect of leptomycin B and mutant forms of Nup214/Can on cellular mRNA export (Bogerd et al. 1998). In contrast, high levels of the CTE can inhibit cellular mRNA export selectively (Pasquinelli et al. 1997; Saavedra et al. 1997), suggesting that cellular mRNA and CTE RNA export must share at least some factor(s) in common.

Efforts to identify a cellular cofactor for CTE-dependent nuclear RNA export have led to the proposal of two candidate human proteins. The Tap protein, first identified as a potential cofactor for the Tip oncogene encoded by Herpes Virus Saimiri (Yoon et al. 1997), has been shown to bind the CTE specifically in vitro and to facilitate CTE function in injected Xenopus oocytes (Grüter et al. 1998). The fact that Tap displays significant homology to Mex67p, a protein that has been proposed to be essential for nuclear mRNA export in yeast cells (Segref et al. 1997), makes Tap an attractive candidate CTE cofactor. A second possible CTE cofactor, RNA helicase A, was also reported to bind the CTE specifically in vitro and was shown to shuttle between the cell nucleus and cytoplasm, a general characteristic of nuclear export factors, and to colocalize with the CTE in expressing cells (Tang et al. 1997). Finally, Pasquinelli et al. (1997) have reported that functional forms of the CTE associate with two nuclear proteins of $\sim 190$ and 85 kD specifically, sizes that would appear inconsistent with either Tap or RNA helicase A.

In this paper, we report the identification of an avian cell line that is nonpermissive for MPMV CTE function and show that coexpression of human Tap rescues this defect completely. Using this cell-based assay for Tap function, in combination with in vivo and in vitro RNAbinding assays, we define a novel RNA-binding motif in Tap and show that this sequence is necessary but not sufficient to mediate Tap activity. By microinjection of a series of mutant recombinant Tap proteins into human cells, we have mapped novel nuclear localization signal (NLS) and NES sequences in the Tap protein. The NES, which lies outside the Tap RNA-binding domain, is critical for Tap function and, indeed, mutants of Tap lacking a functional NES act as dominant-negative inhibitors of wild-type Tap function. Finally, we demonstrate that the
Tap NES, although it does not act via the Crm1 nuclear export factor, can nevertheless be replaced functionally by the Rev NES. Together, these data fully validate Tap as the first known human sequence-specific nuclear mRNA export factor and define novel RNA binding and NES sequences that are critical for Tap function.

\section{Results}

Human Tap has been reported previously to be a 559 amino acid protein with a predicted size of $\sim 63 \mathrm{kD}$ (Yoon et al. 1997), and this was the size of the Tap protein utilized in the previous report that proposed Tap as a CTE cofactor (Grüter et al. 1998). However, recently deposited rat and mouse Tap cDNA sequences, although highly homologous to the human sequence, predict a Tap protein of 618 amino acids in length because of a 59 amino acid amino-terminal extension (Tannoch et al. 1998). We therefore cloned 5'-flanking sequences adjacent to the published human Tap initiation codon and observed that this open reading frame also extends $5^{\prime}$ to the published sequence, in this case predicting a 60 amino acid extension that is $92 \%$ identical to the deposited mouse Tap sequence. Preliminary Western analysis has confirmed that the endogenous human Tap protein migrates at $\sim 70 \mathrm{kD}$, the predicted size encoded by this extended Tap open reading frame (data not shown). Whereas most experiments were therefore performed utilizing the longer ' $1 / 619$ ' form of Tap, we have also included the previously published ' $61 / 619$ ' form of Tap in many experiments as we cannot presently exclude the possibility that this shorter form of Tap also exists in vivo. The extended human Tap coding sequence has been deposited with GenBank (accession no. AF112880).

\section{Tap binds the CTE specifically in vivo}

The MPMV CTE RNA export sequence has been shown to fold into an RNA stem-loop structure containing two internal 12-nucleotide loops, designated loops A and B, that form perfect $180^{\circ}$ mirror images of one another (Fig. 1) (Tabernero et al. 1996; Ernst et al. 1997). Both loops are essential for CTE function in primate cells, whereas flanking helical sequences as well as the apical loop appear to largely serve a structural role. In contrast, a single internal CTE loop is sufficient to mediate nuclear RNA export on Xenopus oocytes (Pasquinelli et al. 1997; Tabernero et al. 1997; Grüter et al. 1998). It has remained unclear whether this apparent discrepancy reflects a real difference between cultured primate cells and Xenopus oocytes or is instead caused by a difference in the export assays used in these distinct in vivo systems.

Although Tap has been shown previously to bind the CTE specifically in vitro (Grüter et al 1998), we wished to confirm that this interaction could also occur in a primate cell nucleus and, more specifically, whether Tap binding would require one or both internal loops. The mammalian RNA-protein binding assay used to examine this question takes advantage of the unique ability of 


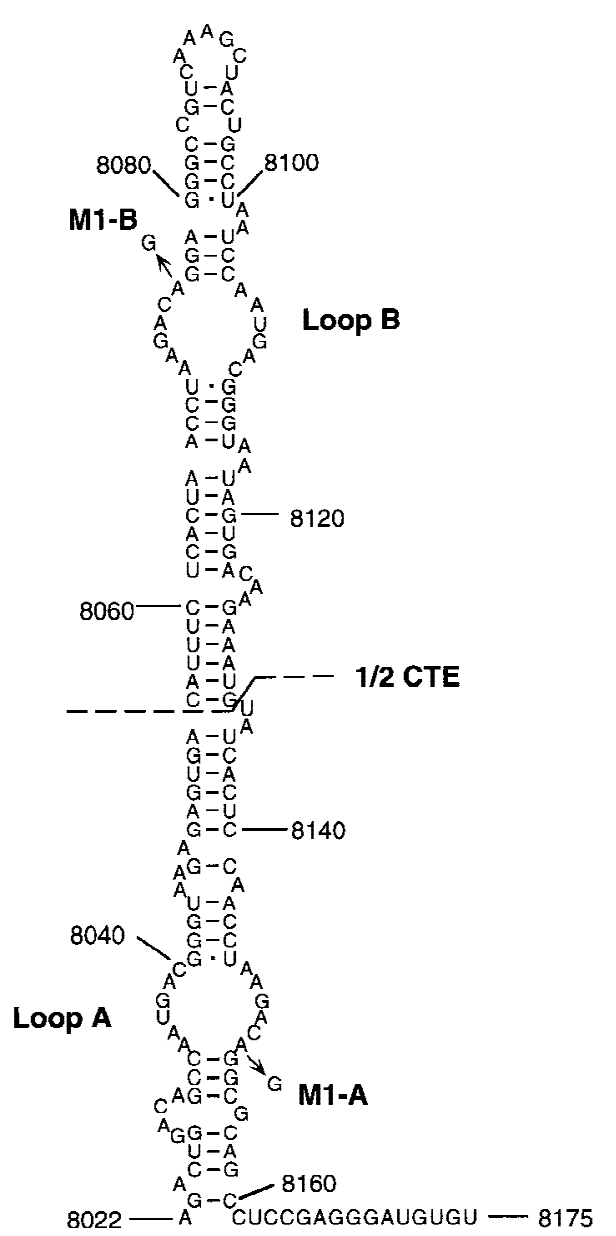

Figure 1. Predicted structure of the MPMV CTE RNA element. The CTE is believed to fold into a helical RNA structure that presents two identical RNA loops, designated loop A and loop B, that serve as specific protein binding sites. The fulllength CTE used here is the MPMV sequence indicated. The extent of the 1/2 CTE RNA sequence is shown, as is the location of the M1 point mutants. Sequence coordinates refer to the full-length MPMV genome and are as defined by Ernst et al. (1997).

the HIV-1 Tat protein to activate HIV-1 long terminal repeat (LTR)-dependent transcription via an RNA target (Selby and Peterlin 1990; Tiley et al. 1992; Blair et al. 1998). Normally, Tat interacts with an RNA stem-loop structure, termed TAR, found just $3^{\prime}$ to the HIV-1 LTR transcription start site. However, Tat can also activate the LTR when it is recruited to a heterologous RNA target, substituted in place of TAR, after fusion to the appropriate RNA-binding protein (Selby and Peterlin 1990; Tiley et al. 1992). We therefore substituted wildtype or mutant forms of the CTE in place of the TAR element present in the HIV-1 LTR and measured the level of activation observed upon cotransfection of human $293 \mathrm{~T}$ cells with a plasmid expressing a Tat-Tap fusion protein.

As shown in Figure 2A, an indicator construct containing the complete MPMV CTE substituted in place of TAR was effectively activated by the Tat-Tap fusion protein, but not by unfused Tat. As controls for binding specificity, we used mutant forms of the CTE bearing a single base mutation in loop B (M1-B) the equivalent mutation in loop A (M1-A) or a double-loop mutant (M1AB) (Fig. 1). Importantly, all three of these CTE mutants are defective in mediating RNA export in transfected cells (see below). Nevertheless, both the CTE M1-A and the CTE M1-B mutant proved able to interact with the Tat-Tap fusion protein, although less well than the wildtype CTE (Fig. 2A). In contrast, the CTE M1-AB double mutant did not bind Tat-Tap detectably. These data therefore imply that a single intact internal CTE loop can bind Tap specifically in vivo. To confirm this unequivocally, we substituted a half CTE, i.e., the apical 78 nucleotide of the CTE containing loop B only (Fig. 1), in place of the HIV-1 TAR element. As shown in Figure 2A, whereas the wild-type half CTE sequence bound the Tat-Tap fusion protein essentially as effectively as did the full-length CTE, introduction of the M1 mutation blocked this interaction entirely. We therefore conclude that Tap can bind the CTE specifically in the human cell nucleus and that this interaction requires only a single intact internal CTE loop.

Tap contains a novel RNA-binding domain that includes a leucine rich repeat (LRR) motif

We next wished to define the sequences in Tap that are necessary and sufficient for CTE binding. As shown in Figure 2B, the 61/619 form of Tap, thought previously to constitute full-length Tap, retained wild-type in vivo RNA-binding activity. A Tap deletion mutant consisting of residues 61-372 also retained full RNA-binding capacity in the mammalian cell nucleus, whereas residues 80372 retained close to full activity. However, further deletion of residues 80-101 blocked RNA binding entirely. Similarly, the deletion of residues 351-372 resulted in clearly reduced RNA binding, whereas deletion of residues 330-350 abolished binding (Fig. 2B). Therefore, these data map the amino-terminal border of the Tap RNA-binding domain to between residues 80 and 101 and the carboxy-terminal border, for efficient RNA binding, between residues 350 and 371. Mutation of Tap residues 129-131, located within the mapped RNA-binding domain, to alanine (mutant A5) blocked CTE binding by Tap entirely (Fig. 2B). Importantly, all these mutant TatTap fusion proteins retained full Tat activity when tested using an HIV-1 LTR bearing the TAR RNA target (data not shown) and are therefore functionally expressed in the human cell nucleus. Of interest, the mapped Tap RNA-binding domain does not contain an obvious RNAbinding motif but does include the unusual LRR motif first noted by Segref et al. (1997) in their analysis of Mex67p, the yeast homolog of Tap.

To confirm that the Tap-CTE interaction is direct, and not dependent on any cellular cofactor, we also asked whether wild-type or deleted forms of Tap would bind the CTE in vitro. Recombinant Tap proteins were therefore expressed in bacteria as glutathione $S$-transferase (GST) fusion proteins and analyzed for binding to a wild- 

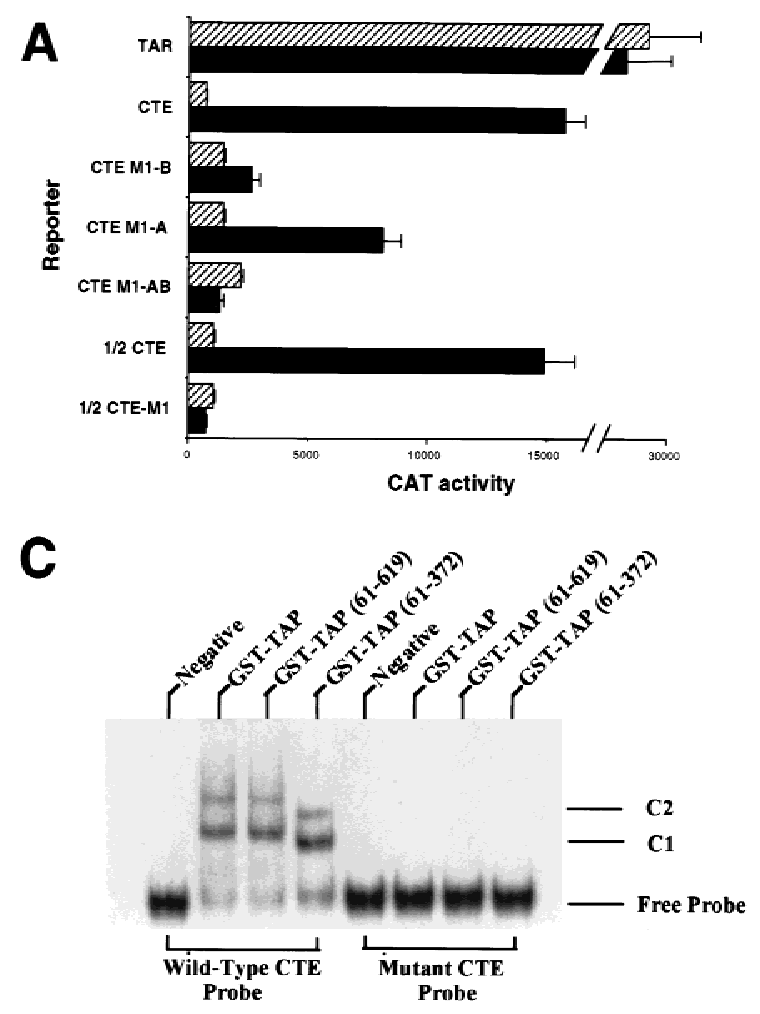
the CTE-binding domain of Tap. The indicated deleted or mutant forms bars) CTE. (C) Tap binds the CTE specifically in vitro. The indicated recombinant proteins, consisting of GST fused to wild-type or deleted forms of Tap, were analyzed for their ability to bind to a wild-type CTE RNA probe, or to a probe bearing a missense mutation (M2) in both CTE loops, using a gel-retardation assay. (C1 and C2) The two discrete retarded RNA-protein complexes observed with the wild-type CTE probe.

type or double loop mutant CTE probe by gel retardation analysis. As shown in Figure 2C, wild-type Tap, as well as Tap residues $61 / 619$ and $61 / 372$, all proved able to bind the wild-type, but not the mutant, CTE in vitro. The formation of two complexes in this analysis may imply binding of Tap to both CTE loops.

\section{Quail cells are rendered permissive for CTE function upon expression of human Tap}

The unequivocal demonstration that Tap is indeed the critical cofactor for CTE function has been difficult because of the lack of a tissue-culture system in which the CTE is inactive. The only system in which the effect of Tap on CTE-mediated RNA export has been reported is Xenopus oocytes, which give enhanced CTE activity upon microinjection of recombinant Tap protein (Grüter et al. 1998). In primate cells, in principle the most relevant system for studying CTE function, we were unable to enhance the biological activity of the CTE by overexpression of Tap (data not shown), possibly implying that the level of expression of Tap is already saturating. Similarly, the CTE proved active in rodent cells and expression of human Tap had little if any positive effect (data not shown). However, as shown in Figure 3A, the quail cell line QCl-3 was not able to support CTE function but was rendered fully permissive for CTE function when inility in $293 \mathrm{~T}$ cells as described in $A$ (Hatched bars) $1 / 2$ CTE-M1; (solid

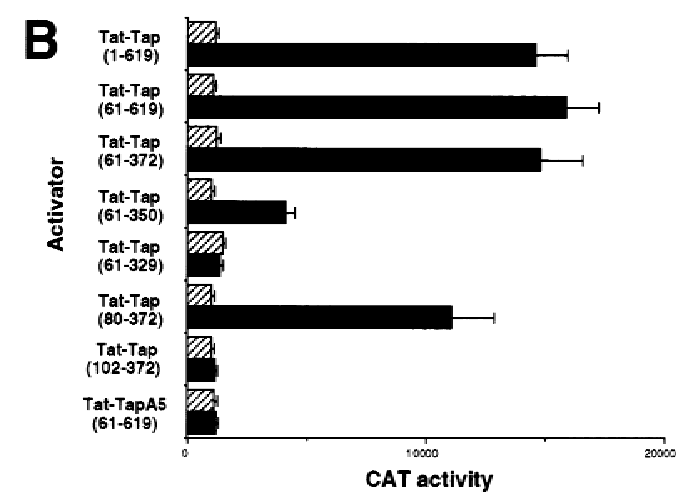

Figure 2. Analysis of the RNA-binding properties of human Tap. (A) Tap binds the CTE specifically in the human cell nucleus. The assay shown relies on the known ability of the HIV-1 Tat protein to activate transcription from the HIV-1 LTR when targeted to an RNA target sequence introduced in place of the viral TAR element. In this case, the TAR element has been replaced by the indicated wild-type or mutant forms of the MPMV CTE in an indicator construct in which expression of the cat indicator gene is controlled by the resultant chimeric HIV-1 LTR promoter. CAT activities observed in transfected human 293T cells, upon expression in trans of wild-type Tat (Hatched bars) or a Tat-Tap (solid bars) fusion protein, are indicated. Human $293 \mathrm{~T}$ cells (35-mm culture) were transfected with $100 \mathrm{ng}$ of the CAT-based indicator construct, 500 $\mathrm{ng}$ of the Tat or Tat-Tap expression plasmid, $1 \mu \mathrm{g}$ of $\mathrm{pBC} 12 / \mathrm{CMV}$ and 25 ng of the $\mathrm{pBC} 12 / \mathrm{CMV} / \beta$-gal internal control plasmid. (B) Definition of

\begin{abstract}
C2) The two discrete retarded RNA-protein complexes observed with
\end{abstract}
human Tap was coexpressed. This experiment utilized the previously described pDM128/CTE indicator plasmid, which encodes the cat indicator gene and wild-type CTE located between functional $5^{\prime}$ and $3^{\prime}$ splice sites (Bogerd et al. 1998; Otero et al. 1998). Because splicing removes the cat open reading frame, efficient CAT expression is dependent on the nuclear export of the unspliced cat mRNA, i.e., on CTE function. As shown elsewhere previously, the wild-type CTE present in pDM128/CTE results in a significant increase in CAT expression, relative to control pDM128 derivatives, when analyzed in transfected primate cells (Bogerd et al. 1998). In contrast, in QCl-3 cells, the presence of the wild-type CTE gives an increase less than or equal to twofold in the level of CAT expression when compared to the defective M1-AB CTE loop mutant (Fig. 3A). However, expression of full-length human Tap results in a dramatic CTE-dependent increase in the level of CAT expression. This activation required a full-length CTE bearing two functional loops, in that the M1-A and M1-B mutants of the full-length CTE, each of which contains one intact and one defective loop (Fig. 1), were not responsive to Tap (Fig. 3B). Similarly, an indicator construct bearing the half CTE sequence (Fig. 1) also failed to respond to Tap in transfected quail cells (Fig. 3B). Whereas these results are identical to the phenotypes of these CTE mutants when analyzed in human cells in the 

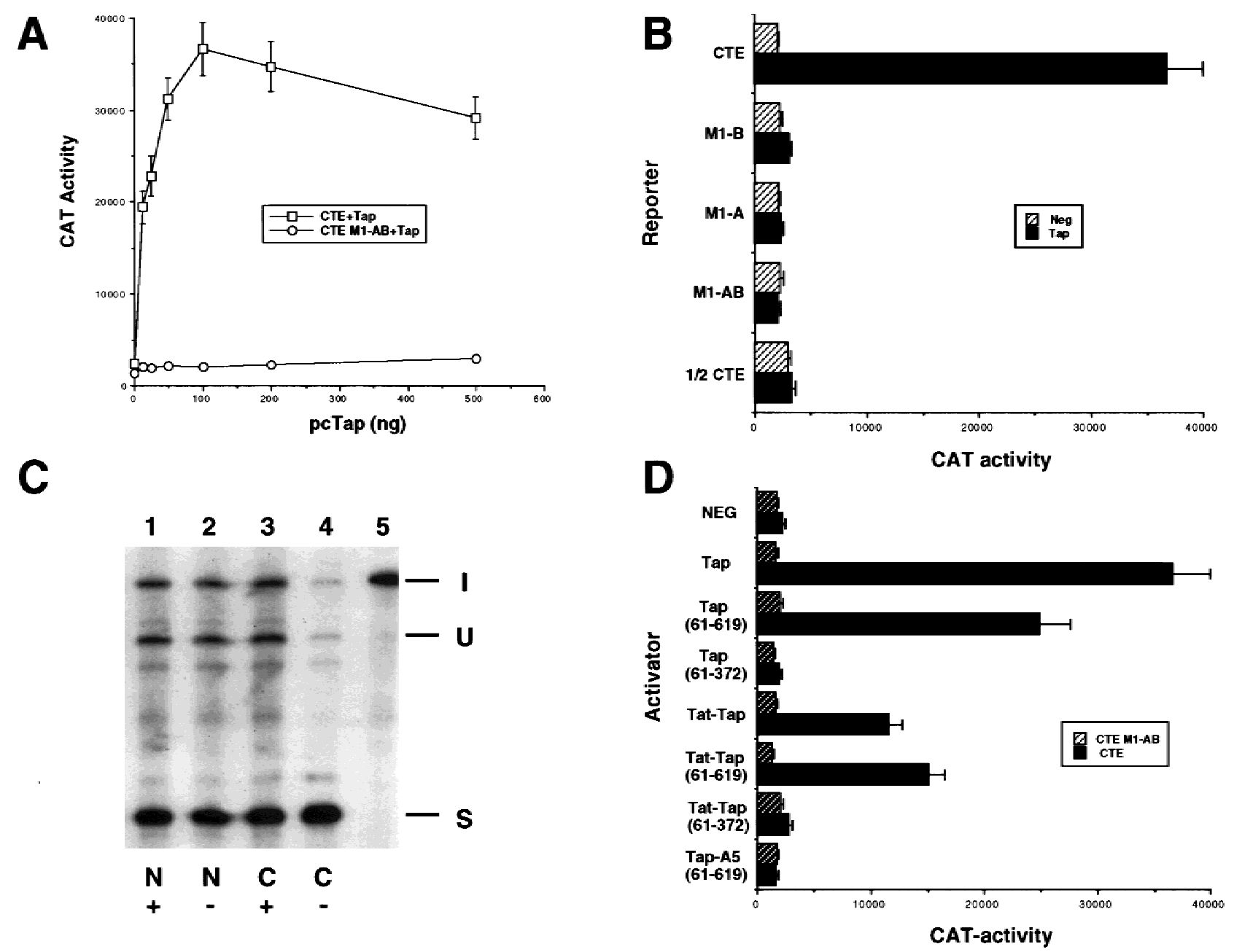

Figure 3. Human Tap induces CTE-dependent nuclear mRNA export in normally nonpermissive quail cells. $(A)$ The quail cell line QCl-3 does not normally support MPMV CTE function, as shown by the nearly equivalent level of activity observed with pDM128/ CTE constructs containing wild-type or defective M1 mutant forms of the CTE. However, cotransfection of an expression plasmid encoding human Tap effectively rescues indicator gene expression from the wild-type, but not mutant, CTE indicator plasmid. QCl-3 cells were transfected with $25 \mathrm{ng}$ of wild-type or M1 mutant forms of the pDM128/CTE indicator plasmid and increasing levels, up to $500 \mathrm{ng}$, of a pBC12/CMV based Tap expression plasmid. Levels of transfected DNA were maintained at a constant level by addition of the parental pBC12/CMV plasmid. ( $\square$ ) CTE + Tap; (O) CTE M1-AB + Tap. (B) The ability of the indicated deleted or mutant forms of the CTE to function in QCl-3 cells expressing human Tap was analyzed as described in $A$, using 100 ng of the pcTap expression plasmid. (Hatched bars) negative; (solid bars) Tap. (C) Levels of expression of the spliced (S) or unspliced (U) RNA encoded by pDM128/CTE, in the cell nucleus (N) or cytoplasm (C) were determined using RNase protection analysis in QCl-3 cells transfected with $100 \mathrm{ng}$ of pDM128/CTE in the presence (+) or absence (-) of $100 \mathrm{ng}$ of the cotransfected pcTap expression plasmid. (I) Input probe. $(D)$ The ability of the indicated wild-type or mutant Tap or Tat-Tap proteins to rescue the biological activity of the CTE in transfected QCl-3 cells was analyzed as described in $A$ and $B$. (Hatched bars) CTE M1-AB; (solid bars) CTE.

absence of cotransfected Tap (data not shown), they contrast with the data in Figure 2A showing that a single intact CTE loop is sufficient for specific Tap binding in vivo.

To confirm that the CTE-dependent increase in CAT expression that is induced by Tap in transfected QCl-3 cells is indeed caused by enhanced mRNA export, we isolated total RNA from the nuclear and cytoplasmic fractions of QCl-3 cells transfected with pDM128/CTE in the presence or absence of coexpressed Tap. As shown in Figure 3C, Tap had little or no effect on the relative level of spliced and unspliced RNA expressed in the nuclear fraction (lanes 1 and 2) but enhanced the expression of the unspliced cat mRNA in the quail cell cytoplasm markedly (c.f. lanes 3 and 4). This is precisely the result expected for a nuclear RNA export factor and highly comparable to data reported previously for CTE or Rev/RRE-dependent mRNA export in primate cells (Malim et al. 1989; Bray et al. 1994).

The Tap RNA-binding motif is not sufficient for Tap function in quail cells

In Figure 2, B and C, we observed that residues 61-372 of 
Tap are fully sufficient for CTE RNA binding. To examine whether they are also sufficient to mediate CTE function, we examined the ability of Tap(61-372) to rescue CTE function in quail cells. As shown in Figure 3D, Tap residues $61-372$ proved entirely inactive. Because the data on Tap RNA binding in the mammalian nucleus shown in Figure 2B utilized a Tat-Tap fusion protein, we also tested the activity of these fused forms of Tap to rescue CTE function, in case Tat was necessary to provide some function, such as an NLS in cis. As shown in Figure 3D, Tat-Tap(61-372), unlike Tat-Tap and TatTap(61-619), also failed to rescue CTE function in quail cells, despite showing wild-type levels of CTE binding in the mammalian cell-based RNA-binding assay (Fig. 2B). The Tap A5 missense mutant, which lacks the ability to bind the CTE (Fig. 2B), also failed to rescue CTE function in quail cells (Fig. 3D) even though it is expressed at comparable levels (data not shown). Therefore, it is apparent that the Tap RNA-binding domain is necessary but not sufficient to mediate CTE-dependent nuclear mRNA export. By analogy to other nuclear RNA export factors, including particularly the HIV-1 Rev protein, we therefore predicted that Tap might contain an essential nucleocytoplasmic transport sequence located outside of the RNA-binding domain (Cullen 1998; Izaurralde and Adam 1998).

\section{Tap contains functional NLS and NES sequences}

To map NLS and NES sequences in the human Tap protein, we used assays described previously that visualize the nucleocytoplasmic transport of recombinant proteins that have been microinjected into the cytoplasm or nucleus of HeLa cells (Herold et al. 1998; Truant et al. 1998). At steady state, the $\sim 70-\mathrm{kD}$ Tap protein localizes to the nucleus of expressing cells (see below) and therefore must contain at least one NLS. As shown previously (Truant et al. 1998), NLS function can be detected readily by expressing a candidate sequence as a GST fusion protein and microinjecting the purified protein into the cytoplasm of cultured cells. After a brief incubation at $37^{\circ} \mathrm{C}$, the localization of the injected GST protein can then be determined by immunofluorescence microscopy, using an anti-GST antibody, and compared to the localization of a coinjected tracer protein that lacks NLS activity, in this case rhodamine-labeled IgG.

Frequently, NLSs are dominant over NESs as indicated by the predominantly nuclear localization of the majority of proteins, such as Rev, that contain both (e.g., PiñolRoma and Dreyfuss 1992; Meyer and Malim 1994). It is therefore not always possible to visualize NES function by simple microinjection of a cell nucleus. Rather, it may be necessary to detect nucleocytoplasmic shuttling, i.e., the combined action of an NLS and an NES. As described previously (Herold et al. 1998; Truant et al. 1998), this can be achieved by microinjecting one nucleus in a binuclear cell with the GST fusion protein being analyzed, together with a rhodamine-labeled tracer protein lacking an NES. After a brief incubation at $37^{\circ} \mathrm{C}$, a protein containing both an NES and NLS will equili- brate between the two resident nuclei, whereas test proteins lacking an NES will, like the tracer, remain confined to the initially injected nucleus.

A series of fusion proteins, consisting of wild-type or mutant forms of Tap linked to GST, were expressed in bacteria, purified, and then analyzed for NLS and NES function as described above. A summary of the data obtained is presented in Figure 4A. Representative key cytoplasmic microinjections are shown in Figure 4B, whereas critical nuclear microinjection data are shown in Figure 4C.

Initially, we attempted to map the predicted Tap NLS. As summarized in Figure 4A, Tap residues 1-619, 61610, and 61-372 all showed clear NLS activity, whereas 80-372 lacked NLS function. This result implied that a Tap NLS was located, at least in part, between residues 61 and 80 and, indeed, Tap residues $61-102$ proved able to induce the exclusively nuclear localization of a GSTTap 61-102 fusion protein (Fig. 4B, panel B). Mutation of this sequence, by replacement of residues 69-71 with alanine, gave rise to a mutant, termed A1, that had lost NLS function entirely (Fig. 4B, panel D).

Analysis of additional GST-Tap fusion proteins showed that Tap residues 1-60 lacked NLS function, as did residues 1-102 bearing the A1 mutation (Fig. 4A). Therefore, there is only one NLS located proximal to the Tap amino terminus. Whereas Tap residues 360-560 also failed to give any detectable NLS activity, we were surprised to observe that Tap residues 540-619 also induced a significant concentration of the GST-Tap 540-619 fusion protein in the nuclei of injected HeLa cells (Fig. 4B, panel F). Mutation of Tap residues 593-595 to alanine reduced significantly, but did not block entirely, the NLS activity of the Tap 540-619 sequence (Fig. 4B, panel H).

Analysis of these same Tap-deletion mutants for NES activity, by microinjection into a single nucleus in binuclear cells, showed that the 61-610 Tap sequence differed from full-length Tap in being unable to shuttle between nucleus and cytoplasm (Fig. 4A). This result implies that the Tap NES must be located, at least in part, either amino-terminal to residue 61 or carboxy-terminal to residue 610. Subsequently, we were able to show that the Tap 540-619 sequence, shown previously to have NLS activity (Fig. 4B, panel F), was also active as an NES (Fig. 4C, panel D). As a result, the GST-Tap 540-619 fusion protein displays nucleocytoplasmic shuttling. This property leads to a steady-state location that is predominantly nuclear, but clearly also partly cytoplasmic, regardless of the initial injection site (compare panel $\mathrm{F}$ in Fig. 4B with panel D in Fig. 4C). In contrast, none of the other GST-Tap fusion proteins, including the 61-102 Tap sequence, which is a potent NLS (Fig. 4B, panel B) displayed any detectable NES activity (Fig. 4C, panel B and data not shown). Of interest, the A17 mutation, which blocked NLS activity only partly (Fig. 4B, panel H) appeared to inhibit the NES function of Tap residues 540-619 entirely (Fig. 4C, panel F). The recombinant rhodamine-labeled tracer protein used in Figure 4C consists of the SV40 T antigen NLS fused to the maltose-binding protein $(\mathrm{MBP})$. This protein retains full NLS function 
A

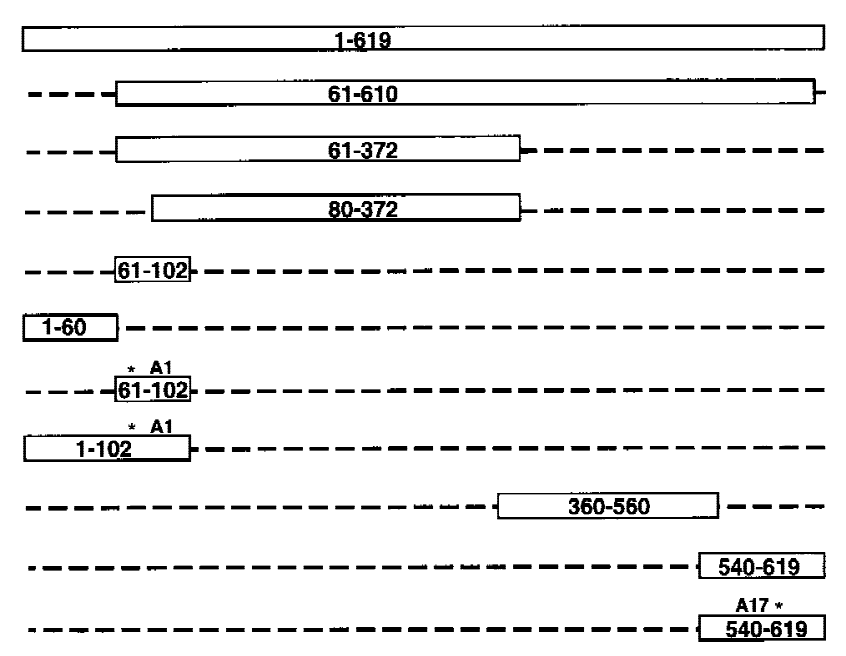

NLS NES

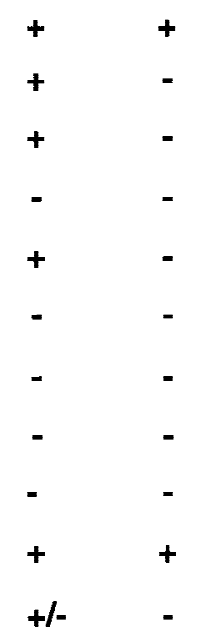

\begin{tabular}{|c|c|c|}
\hline A1 & A5 & A17 \\
\hline $\begin{array}{l}{ }^{67} \text { GPRVRYN } \\
\text { GPAAAYN }\end{array}$ & $\begin{array}{lll}127 & \text { GR KYD } & K^{133} \\
\text { GRAAA KA }\end{array}$ & $\begin{array}{l}591 \text { DNNWDYT } \\
\text { DNAAAYT }\end{array}$ \\
\hline
\end{tabular}

B

61-102

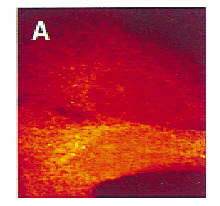

B

anti-

GST

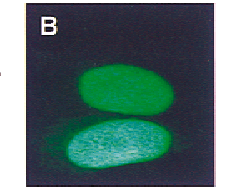

61-102 A1
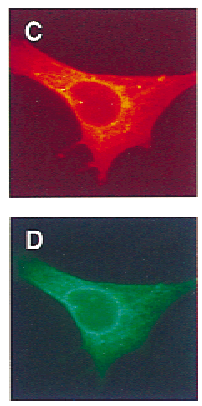

C

MBP

T-NLS

\begin{abstract}
540-619
\end{abstract}
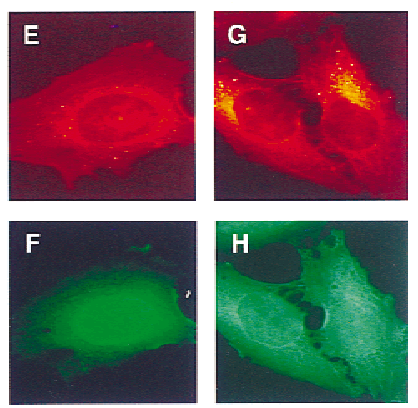

Phase

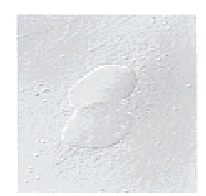

$61-102$

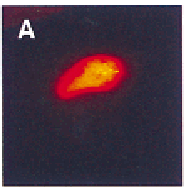

540-619
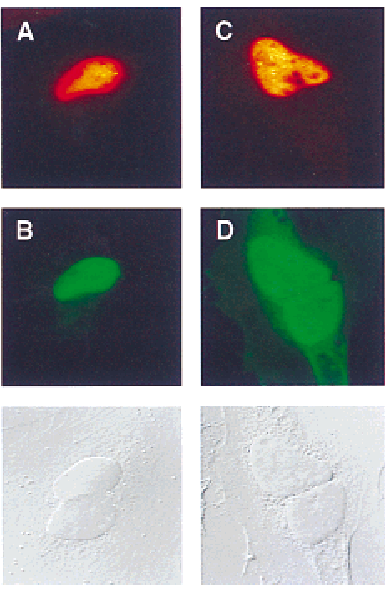

540-619 A17
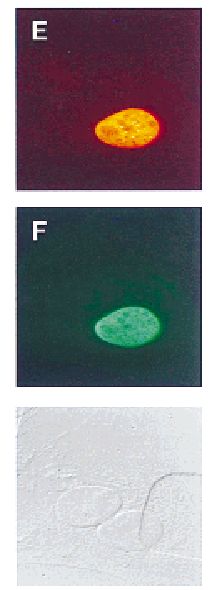

Figure 4. Definition of NLS and NES sequences present in Tap. (A) Summary of the NLS and NES activities displayed by the indicated Tap deletion and missense mutants. Tap proteins were expressed as GST fusion proteins in bacteria and analyzed by cytoplasmic or nuclear microinjection of HeLa cells. The sequence of the A1, A5, and A17 alanine-scanning mutants of Tap reported in this manuscript is indicated at bottom. (B) The indicated recombinant GST-Tap fusion proteins were mixed with rhodamine-labeled IgG and then microinjected into the cytoplasm of HeLa cells. After incubation at $37^{\circ} \mathrm{C}$ for $40 \mathrm{~min}$, the cells were fixed and the subcellular localization of the injected proteins determined by immunofluorescence. $(C)$ Similar to panel B, except that the GST-Tap fusion proteins were microinjected into a single nucleus present in binuclear HeLa cells. Phase photographs are included to show the location of the second nucleus. The rhodamine-labeled tracer protein used here was a recombinant MBP/T-NLS fusion protein.

(Truant et al. 1998) and therefore controls for nuclear uptake of protein inadvertently microinjected into the cell cytoplasm.

The NES present in Tap is critical for CTE-dependent nuclear RNA export

The studies described in Figure 4 mapped an active NLS between Tap residues 61-102 and a second sequence, able to act as both an NLS and NES, between residue 540 and the Tap carboxyl terminus. Both of these signals are located, at least in part, outside of the 80-372 Tap sequence shown to be sufficient for CTE binding (Fig. 2) but inadequate to support CTE function (Fig. 3). The identification of mutations that specifically block the activity of the 61/102 NLS (A1) or the 540/619 NES (A17) (Fig. 4) allowed us to ask whether either or both of these signals are essential for Tap function in the quail cell CTE assay. As shown in Figure 5A, introduction of the A1 mutation into Tap resulted in only a modest drop 
in CTE function. In contrast, inactivation of the 540/619 NES sequence, either by introduction of the A17 missense mutation or by deletion of Tap residues 583-619 (mutant Tap $\Delta$ ), blocked the ability of Tap to rescue CTE
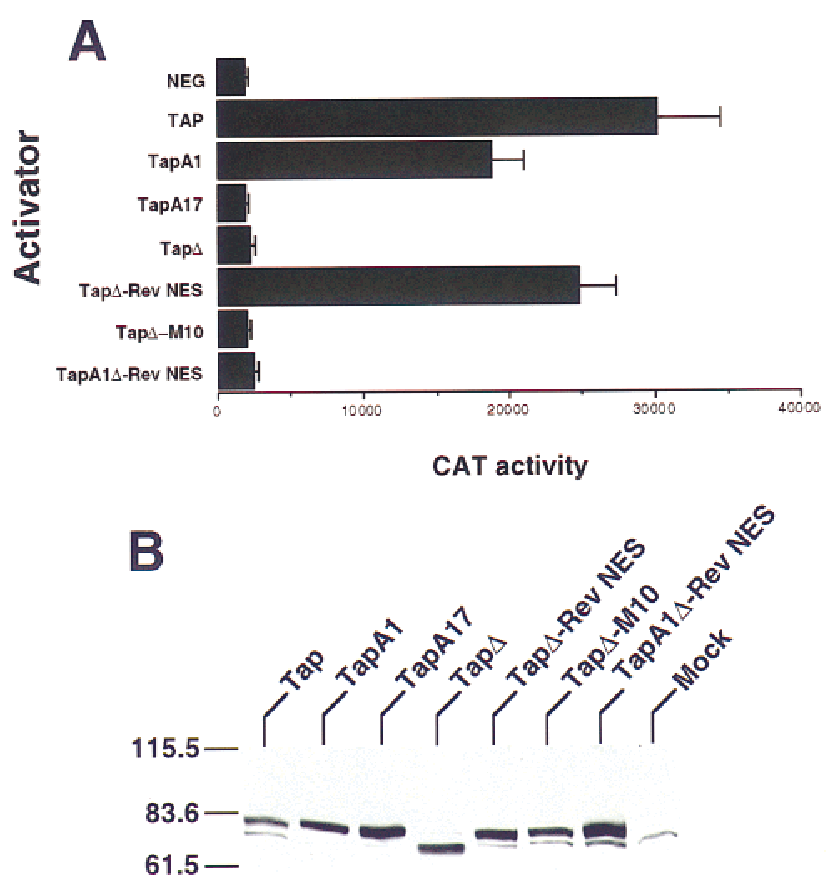

C
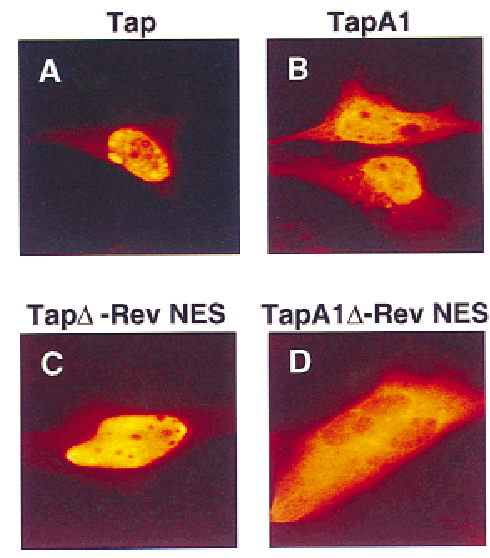

Figure 5. The Tap NES is essential for biological activity but can be substituted with the Rev NES. $(A)$ The indicated Tap missense and deletion mutants, and Tap:Rev chimeras, were tested for biological activity by transfection into QCl-3 cells together with the wild-type pDM128/CTE indicator construct, as described in Figure 2. Whereas all Tap proteins were engineered to express an amino-terminal HA epitope tag, this did not affect their in vivo activity (data not shown). (B) To confirm that the Tap derivatives analyzed for activity in $A$ are expressed, a Western analysis of transfected human $293 \mathrm{~T}$ cells was performed using a monoclonal antibody directed against the amino-terminal HA epitope tag. The relative mobility of molecular size markers is given at left. $(C)$ The subcellular localization of the four indicated Tap derivatives in transfected human HeLa cells was determined by immunofluorescence using an anti-HA epitope tag monoclonal antibody. function completely in transfected quail cells (Fig. 5A). We therefore conclude that the 540/619 NES is essential for Tap function and the 61/102 NLS is not.

Although, as discussed in the introduction, CTE function is not mediated by a Crm 1 -dependent NES equivalent to the leucine-rich NES found in HIV-1 Rev, we nevertheless were interested in whether the Rev NES would be able to rescue the activity of a Tap protein lacking a functional form of the 540/619 NES when introduced in cis. As shown in Figure 5A, attachment of the Rev NES to the carboxyl terminus of the NES-defective Tap $\Delta$ mutant indeed effectively rescued the ability of Tap to support CTE function. In contrast, attachment of the same Rev NES sequence bearing the M10 missense mutation, known to inactivate NES function (Malim et al. 1991; Fischer et al. 1995), failed to rescue this activity. These data therefore confirm that Tap contains a single NES that is critical to support CTE-dependent nuclear RNA export and show that this NES can be replaced functionally by a well-characterized, heterologous NES.

Whereas mutational inactivation of the amino-terminal NLS did not block Tap function, the resultant TapA1 mutant still retained the carboxy-terminal 540/619 sequence that is able to act not only as an NES but also as an NLS (Fig. 4). However, in the Tap $\Delta-$ Rev NES chimera, this shuttling domain has been replaced with the Rev NES, which does not display any NLS activity (Meyer and Malim 1994). Therefore, if the partial activity of the TapA1 mutant indeed reflects the residual NLS activity encoded by the 540/619 Tap-shuttling domain, then introduction of the A1 mutation into Tap $\Delta-$ Rev NES should block Tap function. As shown in Figure 5A, this is exactly what is observed.

To confirm that all the proteins analyzed in Figure 5A are in fact expressed at comparable levels, we performed a Western analysis in transfected human $293 \mathrm{~T}$ cells. The Tap proteins tested in Figure 5A were all expressed with an amino-terminal HA epitope tag, although identical functional data were also observed when otherwise identical untagged proteins were tested (data not shown). This HA tag however allowed the demonstration that all of these proteins, including the inactive Tap variants, are expressed at comparable levels in vivo (Fig. 5B).

We next used immunofluorescence to determine the steady-state subcellular localization, in transfected human HeLa cells, of some of the Tap mutants analyzed in Figure 5A, using an anti-HA monoclonal antibody (Fig. 5C). As expected from the data shown in Figure 4, the wild-type Tap protein gave a predominantly nucleoplasmic localization with nucleolar exclusion (Fig. 5C, panel A). Inactivation of the $61 / 102$ NLS, by introduction of the A1 mutation, resulted in a more diffuse subcellular localization that, while still primarily nuclear, also gave clear cytoplasmic staining (panel B). This localization is therefore similar to that observed upon microinjection of recombinant GST fusion proteins bearing the 540/619 Tap-shuttling sequence (Fig. 4B,C). Replacement of the Tap-shuttling domain with the Rev NES (Tap $\Delta-$ Rev NES) resulted in a subcellular localization essentially in- 
distinguishable from wild-type Tap (Fig. 5C, panel C). However, inactivation of the 61/102 Tap NLS in the context of this Tap/Rev chimera (TapA $1 \Delta-$ Rev NES) resulted in a predominantly cytoplasmic localization clearly distinct from the primarily nuclear localization seen with the Tap A1 mutant (Fig. 5C, cf. B and D). Therefore, these data are consistent with the hypothesis that the $61 / 102$ NLS, although active, is nevertheless functionally redundant with the 540/619 Tap-shuttling domain.

\section{Tap function is not Crm1 dependent}

The biological activity of the Rev NES is dependent on the cellular Crm1 nuclear export factor (Fornerod et al. 1997a; Neville et al. 1997; Stade et al. 1997), whereas CTE function is not affected by agents that inhibit Crm 1 function (Bogerd et al. 1998; Otero et al. 1998; Zolotukhin and Felber 1999). To confirm that the ability of Tap to rescue CTE function in quail cells is also independent of Crm1, we examined the effect of coexpression of a previously described dominant-negative form of the Nup214/Can nucleoporin, termed $\Delta$ Can. It has been shown previously that $\Delta$ Can can bind to Crml and selectively block Rev, but not CTE-dependent nuclear RNA export in transfected primate cells (Fornerod et al. 1997b; Bogerd et al. 1998; Zolotukhin and Felber 1999). As shown in Figure $6 \mathrm{~A}$, expression of $\Delta$ Can in quail cells inhibited significantly the ability of Rev to induce the nuclear export and expression of an RRE containing CAT mRNA yet exerted little effect on the expression of an equivalent CTE-containing mRNA. However, as expected, $\Delta$ Can did inhibit the induction of the identical CTE-dependent CAT mRNA by the Tap $\Delta$-Rev NES protein, in which the Tap NES has been replaced functionally by the Rev NES (Fig. 6A).

\section{Tap mutants lacking a functional NES exert} a dominant-negative phenotype

In the case of the HIV-1 Rev nuclear RNA export factor, mutation of the Rev NES not only blocks Rev function but also results in a dominant-negative phenotype (Malim et al. 1991). This is believed to be because such Rev mutants can compete with wild-type Rev for binding to the HIV-1 RRE RNA target but are then unable to recruit Crm 1 to this RNA. We therefore asked whether the selective inactivation of the Tap NES would give a similar dominant-negative phenotype. As shown in Figure $6 \mathrm{~B}$, this proved to be the case. Specifically, coexpression of either the TapA17 or the Tap $\Delta$ mutant, each of which lacks an NES but retains a complete RNA-binding domain, significantly inhibited the Tap-induced expression of a CTE-containing CAT mRNA. In contrast, coexpression of an inactive Tap mutant (A5) lacking a functional RNA-binding motif but retaining an active Tap NES had at most a modest effect on the level of Tapinduced CTE function. The inhibition of Tap-dependent CTE function by TapA17 or Tap $\Delta$ was specific, in that
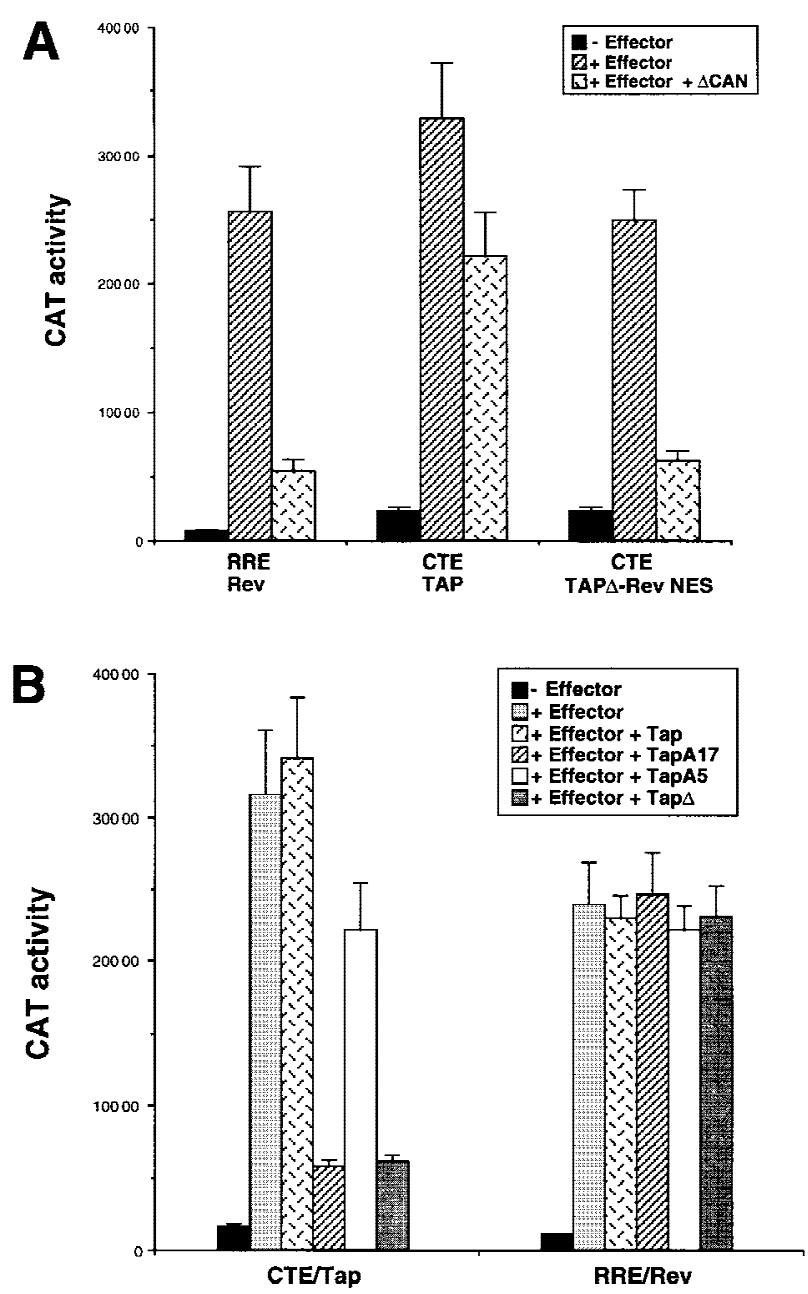

Figure 6. Selective inhibition of either Rev or Tap function in transfected quail cells. QCl-3 cell cultures (35-mm plates) were transfected with $100 \mathrm{ng}(A)$ or $50 \mathrm{ng}(B)$ of a pDM128 indicator plasmid, bearing either the RRE or the CTE RNA target, together with $50 \mathrm{ng}$ of the $\mathrm{pBC} 12 / \mathrm{CMV}$ control plasmid or the pcRev or pcTap expression plasmid. In $A$, the QCl-3 cells were additionally cotransfected with $100 \mathrm{ng}$ of either $\mathrm{pBC} 12 / \mathrm{CMV}$ or of a plasmid expressing the $\Delta$ Can protein, which has been shown to specifically inhibit Crm1 function (Bogerd et al. 1998). In $B$, the QCl-3 cells were instead cotransfected with $500 \mathrm{ng}$ of either $\mathrm{pBC} 12 / \mathrm{CMV}$ or of a plasmid expressing wild-type or the indicated mutant forms of Tap. In each case, induced CAT activity was measured at $\sim 48 \mathrm{hr}$ after transfection.

neither had any detectable effect on the level of activation of an RRE-containing CAT mRNA by the HIV-1 Rev protein (Fig. 6B).

\section{Discussion}

The experiments described in this manuscript validate fully the hypothesis first proposed by Grüter et al. (1998), that MPMV CTE-dependent nuclear mRNA export can be mediated by the human Tap protein. In addition, these experiments identify several important functional 
domains in the Tap protein, including a novel RNAbinding motif and an equally novel NES.

The most convincing, and also most striking, evidence supporting the identification of Tap as a CTE-specific nuclear mRNA export factor is the observation that quail QCl-3 cells, which are normally not permissive for CTE function, are rendered highly permissive upon expression of the human Tap protein (Fig. 3A). Tap is clearly acting in these cells at the level of unspliced RNA export, as was shown directly by analysis of the effect of Tap on nuclear and cytoplasmic RNA expression patterns (Fig. 3C). In these quail cells, Tap therefore gives a phenotype with an unspliced CTE-containing RNA target that is closely comparable to the previously reported effects of HIV-1 Rev on the expression of a similar RREcontaining unspliced RNA (Malim et al. 1989, 1991).

A second line of evidence supporting the identification of Tap as a CTE-specific cofactor is the demonstration that Tap can bind the CTE specifically in the nucleus of human cells (Fig. 2A) and that this interaction is blocked by the introduction into the two CTE loops of single nucleotide missense mutations that also inhibit CTE function. Of interest, however, although CTE binding by Tap in vivo requires only a single intact loop (Fig. 2A), CTE function in both primate cells and in Tap-expressing quail cells clearly requires that both CTE loops remain intact (Fig. 3B) (Tabernero et al. 1996; Ernst et al. 1997). Although the basis for the discrepancy between the CTE sequences required for CTE function versus Tap binding in primate and quail cells remains unclear, it is interesting to note that the nuclear RNA export activity of the HIV-1 Rev and Human T-cell leukemia virus Rex nuclear RNA export factors has also been shown to be dependent on the recruitment of multiple Rev or Rex molecules to their respective RNA target (Huang et al. 1991; Malim and Cullen 1991; Gröne et al. 1994).

\section{The Tap RNA-binding domain is necessary}

but not sufficient for nuclear RNA export

The ability to readily assay RNA binding by Tap, either in vivo or in vitro, allowed us to demonstrate that residues $80-372$ of the 619-amino-acid Tap protein are fully sufficient for sequence-specific RNA binding (Figs. 2 and 7). Mutation of residues 129-131 in Tap mutant A5 blocked RNA binding by Tap (Fig. 2B) as did a number of other alanine-scanning mutants targeted between residues 129 and 347 (data not shown). We therefore con-

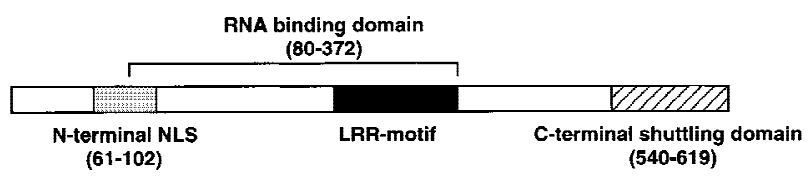

Figure 7. Domain organization of the human Tap nuclear mRNA export factor. The relative location of the RNA-binding domain, amino-terminal NLS and carboxy-terminal nucleocytoplasmic shuttling domains of Tap defined in this paper are shown schematically. clude that Tap contains a novel, sequence-specific RNAbinding motif, the only striking feature of which is an LRR that is also conserved in the yeast homolog of Tap (Segref et al. 1997).

Although RNA binding by Tap proved, as expected, to be required for Tap function, it was not sufficient (Fig. 3D). Based on the precedent of the HIV-1 Rev nuclear RNA export factor this result was not a surprise. Specifically, whereas Rev contains an RNA-binding motif that is essential for RRE-dependent RNA export, Rev activity is also dependent on the Rev NLS and, particularly, the Rev NES (Malim et al. 1991; Fischer et al. 1995). Using previously described protein microinjection assays in HeLa cells, we were able to map an NLS in Tap to between residues 61 and 102 and a nucleocytoplasmicshuttling sequence, that displayed both NLS and NES activity, to between residues 540 and 619 , that is, at the Tap carboxyl terminus (Figs. 4,7). Whereas inactivation of the 61/102 NLS reduced Tap function only modestly, inactivation of the 540/619 NES sequence blocked the ability of Tap to rescue CTE function in QCl-3 cells entirely (Fig. 5A). The demonstration that Tap contains both an NLS and an NES, and is therefore able to shuttle between the nucleus and cytoplasm of expressing cells, represents a third line of evidence supporting the identification of Tap as a nuclear RNA-export factor, given that nucleocytoplasmic shuttling appears to be a general characteristic of both nuclear import and nuclear export factors (Piñol-Roma and Dreyfuss 1992; Meyer and Malim 1994; Izaurralde and Adam 1998; Truant et al. 1998).

\section{Tap function requires both NLS and NES function}

Although the inactivation of the carboxy-terminal NES in Tap by the A17 missense mutation blocks Tap function, it could be argued that this phenotype is caused by an effect of A17 on another, unknown property of Tap. To demonstrate unequivocally that Tap function in fact requires an NES, we therefore substituted the well-characterized Rev NES, or the mutant M10 form of the Rev NES, for the Tap 540/619 NES/NLS and asked whether this substitution would specifically rescue Tap function (Malim et al. 1991; Fischer et al. 1995). As shown in Figure $5 \mathrm{~A}$, this proved to be the case, that is, the Tap $\Delta-$ Rev NES chimera was able to induce CTE function in QCl-3 cells, whereas the similar Tap $\Delta-$ M10 protein was not.

If mutation of the carboxy-terminal Tap NES does not inhibit CTE RNA binding in vivo, as indicated by the data presented in Figure 2B, then mutants of Tap lacking a functional NES, such as A17 and Tap $\Delta$, should compete with wild-type Tap for CTE binding but should then be unable to mediate CTE-dependent RNA export, that is, they should act as specific competitive inhibitors of Tap function. As shown in Figure 6B, Tap $\Delta$ and TapA17 indeed proved able to inhibit specifically Tap/CTE-dependent, but not Rev/RRE-dependent, nuclear RNA export. The Tap A17 and Tap $\Delta$ mutants are therefore reminiscent of the dominant-negative M10 mutant of HIV-1 
Rev, which also lacks an active NES yet retains a functional RNA-binding motif (Malim et al. 1991).

Mutation of the 61/102 Tap NLS did not block Tap function, whereas substitution of the 540/619 Tap NES/ NLS sequence with the Rev NES, which lacks any NLS activity, also permitted Tap function (Fig. 5A). Therefore, it appeared probable that these two Tap NLSs are, at least in part, functionally redundant. As predicted from this hypothesis, mutation of the 61/102 Tap NLS in the context of the Tap $\Delta-$ Rev NES chimera, in which this NLS is the only one present, blocked Tap function entirely. Also consistent with the hypothesis that both the 61/102 and the 540/619 Tap NLS are active in the context of the full-length Tap protein is the observation that inactivation of the 61/102 NLS, in an otherwise wildtype Tap context, results in only a partial redistribution of Tap from the nucleus to the cytoplasm (Fig. 5C, panel B). In contrast, mutation of this NLS in the context of the Tap $\Delta$-Rev NES results in a predominantly cytoplasmic localization (Fig. 5C, panel D). Together, these data therefore confirm that Tap contains an NES and two NLS sequences (Fig. 7) and demonstrate that Tap function requires an intact NES and at least one intact NLS.

\section{The Tap NES and NLSs belong to novel functional classes}

It has been proposed that CTE function is independent of Crm1, the cellular export factor that mediates the function of the leucine-rich NES found in HIV-1 Rev (Fornerod et al. 1997a; Neville et al. 1997; Stade et al. 1997; Bogerd et al. 1998; Otero et al. 1998; Zolotukhin and Felber 1999). This result is confirmed in Figure 6A, which shows that $\Delta$ Can, a dominant-negative form of the nucleoporin Nup214/Can that specifically inhibits Crm1 function, can inhibit the activity of Rev, and of the Tap $\Delta-$ Rev NES chimera in quail cells but not the activity of the wild-type Tap protein. Therefore, the protein target for the Tap NES is not Crm1. In fact, we have been unable to demonstrate an interaction between the Tap NES and either Crm1, or the distinct nuclear export factor Cas, under conditions (Herold et al. 1998) in which a Rev NES-Crm1 or an Importin $\alpha$ NES-Cas interaction can be detected readily (data not shown). In this context, it is of interest to note that Mex67p, the yeast homolog of Tap, has been shown to associate with Mtr2p and this interaction has been proposed to target Mex67p to the nuclear pore (Santos-Rosa et al. 1998). Although Mtr2p, like Mex67p, has been proposed also to play an essential role in mediating nuclear mRNA export in yeast cells (Kadowaki et al. 1994), no human Mtr2p homolog is presently known. Clearly, however, the future identification of the protein target for the Tap NES will be critical for understanding both the mechanism of action and the normal biological role of Tap.

In addition to the Tap NES, the Tap 61/102 NLS and the NLS activity located between Tap residues 540 and 619 also appear novel. Specifically, neither sequence contains a stretch of lysine residues equivalent to that seen in basic, Importin $\alpha$-dependent NLSs, such as the
SV40 T antigen NLS (Izaurralde and Adam 1998). In fact, the 61/102 NLS is devoid entirely of lysine. Furthermore, preliminary experiments have not revealed any interaction between either the 61/102 NLS or the 540/ 619 NLS and Importin $\alpha$ under conditions (Herold et al. 1998) in which an interaction between Importin $\alpha$ and several basic NLSs is readily detectable (data not shown). It is therefore likely that these two NLS sequences act via other cytoplasmic NLS receptors, a number of which have been proposed or shown to exist (Izaurralde and Adam 1998).

We have not, at this point, been able to clearly mutationally segregate the NLS and NES activities of the Tap 540/619 nucleocytoplasmic-shuttling motif. At least three other sequences that display both NES and NLS activity have been reported, i.e., the so-called M9 NES/ NLS found in hnRNP A1 (Michael et al. 1995) and nucleocytoplasmic-shuttling domains observed in hnRNP K (Michael et al. 1997) and HuR (Fan and Steitz 1998). At least in the case of the M9 sequence, it has proven impossible to segregate NES from NLS function using an exhaustive mutational approach (Bogerd et al. 1999). Although inspection of these three other shuttling domains does not reveal any obvious homology to the Tap 540/ 619 sequence, it nevertheless remains possible that one or more of these shuttling domains acts via the same NES and/or NLS receptor.

\section{Implications for cellular $m R N A$ export}

A number of observations suggest that heterogeneous nuclear ribonucleoproteins (hnRNPs), a group of highly expressed proteins that associate with nuclear mRNA and pre-mRNA, are key participants in the nuclear export of mRNAs (for reviews, see Izaurralde and Adam 1998; Stutz and Rosbash 1998). In mammalian cells, the best-studied hnRNP is hnRNP Al, a protein that binds to mRNAs in the nucleus and remains bound to mRNA during the process of nucleocytoplasmic transport (Piñol-Roma and Dreyfuss 1992). Once in the cytoplasm, hnRNP A1 is released and returns to the nucleus. As noted above, nucleocytoplasmic shuttling by hnRNP A1 is not passive, in that this protein displays both NLS and NES activity (Michael et al. 1995). The importance of the hnRNP A1 NES in mediating mRNA export is supported by studies in Xenopus oocytes showing that microinjection of hnRNP A1 variants containing a functional NES can selectively inhibit mRNA nuclear export (Izaurralde et al. 1997).

In yeast, a protein termed Npl3p, which may serve as the functional homolog of hnRNP A1, also associates with nuclear mRNA and shuttles between the nucleus and cytoplasm (Lee et al. 1996). Whereas loss of Npl3p function results in an inhibition of mRNA export, it is also true that blocking mRNA export inhibits Npl3p nucleocytoplasmic shuttling. This finding confuses the issue of which molecule is the cargo and which is the carrier and also distinguishes Npl3p from mammalian hnRNP A1, which contains an NES that functions independently of mRNA binding (Michael et al. 1995). Nev- 
ertheless, these observations, in total, provide persuasive evidence in favor of the hypothesis that hnRNP-like proteins are key mediators of mRNA export.

Given this context, it is at present difficult to understand how Tap fits into the nuclear export of cellular mRNAs. Whereas CTE-dependent mRNA export is clearly dependent on Tap, this requires a specific protein:RNA interaction that can not be directly relevant to global mRNA export. On the other hand, if Tap bound all mRNAs promiscuously, then the CTE should not be required to target Tap to the MPMV genomic RNA. Therefore, if Tap is indeed involved in global mRNA export, this is likely to be at a late, regulated step in this pathway that is mediated either by nonspecific mRNA binding by Tap or, perhaps, by recruitment of Tap to mRNAs by prebound proteins such as hnRNPs. The observation that overexpression of the CTE in Xenopus oocytes selectively blocks mRNA export, and that this inhibition can be relieved by injection of recombinant Tap protein (Pasquinelli et al. 1997; Grüter et al. 1998), taken together with the finding that inactivation of the yeast Tap homolog Mex67p can selectively inhibit mRNA export (Segref et al. 1997), strongly suggests a role for Tap in the export of cellular mRNAs lacking a CTE equivalent. The unequivocal demonstration, in this paper, that Tap is a nuclear mRNA export factor, together with the identification of critical domains in Tap required for nuclear RNA export, should greatly facilitate future efforts to prove or refute this hypothesis.

\section{Materials and methods}

\section{Plasmid construction}

All eukaryotic expression plasmids utilized in this work are based on pBC12/CMV (Malim et al. 1991), which also serves as a negative control. The following $\mathrm{pBC} 12 / \mathrm{CMV}$-based expression plasmids have been described previously: pcTat, pcRev, $\mathrm{pBC} 12 / \mathrm{CMV} / \beta$-gal (Tiley et al. 1992) and pc $\Delta \mathrm{CAN}$ (Bogerd et al. 1998); the HIV-1 Rev indicator construct pDM128/CMV (Hope et al. 1990; Malim et al. 1991), and an equivalent construct containing the MPMV CTE (pDM128/CTE) in place of the RRE element (Bogerd et al. 1998); a reporter plasmid containing the cat indicator gene under the control of the wild-type HIV-1 LTR (pTAR/CAT) (Tiley et al. 1992).

A DNA sequence encoding amino acids 61-619 of human Tap was amplified by PCR from pHA-Tap (Yoon et al. 1997) and cloned between the NcoI and XhoI sites of pBC12/CMV to generate pcTap(61-619). The 60-amino acid amino-terminal extension of the coding sequence of Tap was PCR amplified from a Clontech human T-cell cDNA library using a primer that introduced an NcoI site at the $5^{\prime}$ end of the coding sequence and a second primer complementary to the central part of the Tapcoding region. The resultant PCR product was digested with NcoI and Bsp120I, which recognizes a unique site in the Tapcoding region, and inserted into the pcTap(61-619) plasmid digested with NcoI and Bsp120I. The resultant pcTap plasmid encodes the full-length human Tap protein. Truncated forms of Tap were generated by PCR and cloned into the NcoI and XhoI site of pBC12/CMV. The full length tat gene was amplified by PCR with primers that introduced flanking $N c o$ I sites and then ligated into NcoI-digested pcTap series plasmids to generate plasmids that express chimeric proteins consisting of Tat fused to full-length or mutant Tap proteins. Sequences encoding amino acids $69-71,129-131$, and 593-595 of Tap in pcTap or pcTat-Tap(61-619) were mutated using a Quickchange kit (Stratagene) to GCGGCCGCC, which encodes triple alanine, to generate pcTapA1, pcTapA5, pcTapA17, and pcTat-Tap/61619|A5.

A unique $\mathrm{XhoI}$ site encoding amino acids 582-583 was mutated into two consecutive stop codons (TGATAA) in pcTap to generate $\operatorname{pcTap} \Delta$. A DNA fragment with flanking Xhol sites, harboring the coding sequence of the Rev NES (PVPLQLPPLERLTLDCNEDCG) followed immediately by a stop codon, was inserted in-frame into this $X h o I$ site to generate pcTap $\Delta-R e v$ NES. The Al alanine substitution mutation, at amino acids 69-71 of Tap, and the previously described (Malim et al. 1991) M10 mutation of the Rev NES were introduced into pcTap $\Delta-$ Rev NES using Quickchange to generate pcTapA1-Rev NES, and pcTap-M10, respectively. A DNA fragment encoding a triple HA epitope tag, with flanking $N c o$ I sites, was inserted into the NcoI site present at the $5^{\prime}$ end of the Tap coding sequence of all pcTap series plasmids to generate the epitopetagged Tap expression plasmids used in Figure 5.

CTE fragments with flanking BamHI sites at each end were PCR-amplified and inserted into the BgIII site of pDM128/CMV (Malim et al. 1991), as well as the BamHI site present in the in vitro transcription plasmid pGEM-3fZ. Mutant/variant CTE constructs (Fig. 1) were generated by using the Quickchange kit (Stratagene). The M2 CTE mutant has AGU substituted for residues 8073-GAC-8075 and 8149-GAC-8151 in both CTE loops. The half CTE includes the apical 78 nucleotides of the CTE. Reporter plasmids for mammalian CTE-binding assays were generated by replacing the TAR element in pTAR/CAT with wild-type or mutant/variant CTE elements. CTE fragments were PCR amplified from pDM128/CMV based CTE constructs using primers containing unique BgIII and SacI sites and inserted into pTAR/CAT digested with the same enzymes.

Full-length or truncated forms of Tap were PCR-amplified and inserted into the EcoRI and XhoI sites present in the bacterial GST fusion protein expression vector pGEX-4T-1. Alanine substitution mutations were introduced into sequences encoding amino acids 69-71 (mutant A1), or 593-595 (mutant A17) of Tap in pGST-Tap(61-102) and pGST-Tap(540-619) using the Quickchange kit (Stratagene), to generate pGST-Tap(61-102)A1 and pGST-Tap(540-619)A17.

The DNA sequence in pDM128/CMV that flanks the 3' splice site, which is derived from pgTAT (Malim et al. 1989), was PCR amplified with primers containing EcoRI and KpnI sites and cloned into these two sites in pGEM3fZ (Promega) to generate the in vitro transcription plasmid pT7-RPA. In vitro transcription of pT7-RPA using T7 polymerase generated an antisense RNA fragment, that starts at nucleotide 3450 of pgTAT and then extends through the predicted 3 ' splice site, at nucleotide 3349, to end at nucleotide 3293.

\section{Cell culture and transfection}

Human 293T and HeLa cells and quail QCl-3 cells were maintained as described previously (Cullen et al. 1983; Bogerd et al. 1998) and transfected using lipofectamine (Life Technologies), GenePORTER transfection reagent (Gene Therapy System) or DEAE-dextran (Cullen et al. 1983), respectively. All transfections were performed on cell cultures in $35-\mathrm{mm}$ plates. Levels of DNA used in each transfection experiment are given in the relevant figure legend, with $\mathrm{pBC} 12 / \mathrm{CMV} / \beta$-gal included as an internal control. In all transfection experiments, CAT enzyme levels were determined $\sim 48 \mathrm{hr}$ after transfection as described previously, and normalized to the level of $\beta$-gal activity present 
in the cell lysate (Bogerd et al. 1998). Western blot analysis of protein expression levels in transfected 293T cells and indirect immunofluorescence analysis of transfected HeLa cells, were performed using a monoclonal mouse anti-HA tag antibody (Roche Molecular Biochemicals) essentially as previously described (Fridell et al. 1996; Hua et al. 1997).

\section{Gel-shift analysis}

GST fusion proteins, containing full-length or truncated forms of Tap, were expressed in bacteria and purified on glutathione affinity resin as described previously (Bogerd et al. 1999). The wild-type and M2 mutant CTE RNA probes were labeled with $\left[\alpha{ }^{32}\right.$ P]CTP using the Riboprobe in vitro transcription system (Promega) and the total isotope incorporation determined by scintillation counting after column purification. The binding reaction was carried out with $\sim 10^{4} \mathrm{cpm}(\sim 0.1 \mathrm{ng})$ of the probe and $25 \mathrm{ng}$ of GST-Tap fusion protein in $20 \mu \mathrm{l}$ of binding buffer [150 mM KCl, $10 \mathrm{~mm}$ HEPES (pH 7.6), 0.5 mM EGTA, 2 mM $\mathrm{MgCl}_{2}, 1 \mathrm{~mm}$ DTT, and $10 \%$ glycerol] containing $4 \mu \mathrm{g}$ of rRNA and $1 \mu \mathrm{g}$ of yeast tRNA. Binding was allowed to proceed for 20 min at $4^{\circ} \mathrm{C}$ and the reaction products resolved on a $5 \%(40: 1)$ native polyacrylamide gel and visualized by autoradiography.

\section{HeLa cell microinjection}

HeLa cells were maintained and microinjected as described previously (Herold et al. 1998; Truant et al. 1998). Briefly, 2 days before microinjection, HeLa cells were seeded onto CELLocate microgrid coverslips (Eppendorf Scientific) at a density of $2 \times 10^{5} / 35-\mathrm{mm}$ dish. The test proteins (final concentration in PBS $\sim 2 \mu \mathrm{g} / \mu \mathrm{l}$ ) were coinjected with a rhodamine-conjugated goat IgG (in import assays; Cappel Laboratories) or with a previously described (Truant et al. 1998) tetramethylrhodamine isothiocyanate-conjugated MBP-TNLS fusion protein (for export assays) as tracers (final concentration $1.5 \mu \mathrm{g} / \mu \mathrm{l}$ ) to verify the site of injection. After injection, cells were incubated at $37^{\circ}$ for $40 \mathrm{~min}$ and then fixed with 3\% paraformaldehyde in PBS. The GST fusion proteins were then visualized by indirect immunofluorescence using a polyclonal affinity-purified rabbit anti-GST antibody and fluorescein isothiocyanate-conjugated donkey anti-rabbit antiserum. The subcellular localization of the injected proteins was visualized using a Leica DMRB fluorescence microscope at a $100 \times$ magnification.

\section{RNA isolation and ribonuclease protection assay}

Quail QCl-3 cells (six-well 35-mm plates) were transfected with $100 \mathrm{ng}$ of pcTap or pBC12/CMV, $25 \mathrm{ng}$ of pDM128/CTE, and $400 \mathrm{ng}$ of pBC12/CMV. Nuclear and cytoplasmic RNAs were fractionated from QCl-3 cells at $72 \mathrm{hr}$ after transfection, and purified by SV Total RNA Isolation System (Promega) and RNeasy Cytoplasmic RNA Isolation Kit (Qiagen), respectively. Ribonuclease protection assay (RPA) analysis was performed using the Hyspeed RPA kit (Ambion) following the manufacturer's protocol. The RNA probe used in this assay was generated by in vitro transcription of plasmid pT7-RPA linearized at the HindIII site, using the Riboprobe kit (Promega). The input probe is 208 nucleotides in length, with additional tag sequence derived from vector sequence between KpnI and HindIII sites to allow the full-length, input probe to be distinguished from probe fragments rescued by the unspliced and spliced mRNA transcripts, which have a predicted length of 158 and 102 nucleotides, respectively.

\section{Acknowledgments}

We thank Jae Jung for the human Tap cDNA and Hal Bogerd,
Paul Bieniasz, and Elisa Izaurralde for helpful discussions. This research was supported by the Howard Hughes Medical Institute.

The publication costs of this article were defrayed in part by payment of page charges. This article must therefore be hereby marked 'advertisement' in accordance with 18 USC section 1734 solely to indicate this fact.

\section{References}

Blair, W.S., T.B. Parsley, H.P. Bogerd, J.S. Towner, B.L. Semler, and B.R. Cullen. 1998. Utilization of a mammalian cellbased RNA binding assay to characterize the RNA binding properties of picornavirus 3C proteinases. RNA 4: 215-225.

Bogerd, H.P., A. Echarri, T.M. Ross, and B.R. Cullen. 1998. Inhibition of human immunodeficiency virus Rev and human T-cell leukemia virus Rex function, but not mason-pfizer monkey virus constitutive transport element activity, by a mutant human nucleoporin targeted to Crm1. J. Virol. 72: 8627-8635.

Bogerd, H.P., R.E. Benson, R. Truant, A. Herold, M. Phingbodhipakkiya, and B.R. Cullen. 1999. Definition of a consensus transportin-specific nucleocytoplasmic transport signal. $I$. Biol. Chem. 274: 9771-9777.

Bray, M., S. Prasad, J.W. Dubay, E. Hunter, K.-T. Jeang, D. Rekosh, and M.-L. Hammarskjöld. 1994. A small element from the mason-pfizer monkey virus genome makes human immunodeficiency virus type 1 expression and replication Revindependent. Proc. Natl. Acad. Sci. 91: 1256-1260.

Chang, D.D. and P.A. Sharp. 1989. Regulation of HIV Rev depends upon recognition of splice sites. Cell 59: 789-795.

Cullen, B.R. 1998. Retroviruses as model systems for the study of nuclear RNA export pathways. Virology 249: 203-210.

Cullen, B.R., A.M. Skalka, and G. Ju. 1983. Endogenous avian retroviruses contain deficient promoter and leader sequences. Proc. Natl. Acad. Sci. 80: 2946-2950.

Ernst, R.K., M. Bray, D. Rekosh, and M.-L. Hammarskiöld. 1997. Secondary structure and mutational analysis of the masonpfizer monkey virus RNA constitutive transport element. RNA 3: 210-222.

Fan, H.C. and J.A. Steitz. 1998. HNS, a nuclear-cytoplasmic shuttling sequence in HuR. Proc. Natl. Acad. Sci. 95: 15293 15298.

Fischer, U., J. Huber, W.C. Boelens, I.W. Mattaj, and R. Lührmann. 1995. The HIV-1 Rev activation domain is a nuclear export signal that accesses an export pathway used by specific cellular RNAs. Cell 82: 475-483.

Fornerod, M., M. Ohno, M. Yoshida, and I.W. Mattaj. 1997a. CRM1 is an export receptor for leucine-rich nuclear export signals. Cell 90: 1051-1060.

Fornerod, M., J. van Deursen, S. van Baal, A. Reynolds, D. Davis, K.G. Murti, J. Fransen, and G. Grosveld. 1997b. The human homologue of yeast CRM1 is in a dynamic subcomplex with CAN/Nup214 and a novel nuclear pore component Nup88. EMBO J. 16: 807-816.

Fridell, R.A., R.E. Benson, J. Hua, H.P. Bogerd, and B.R. Cullen. 1996. A nuclear role for the fragile $\mathrm{X}$ mental retardation protein. EMBO J. 15: 5408-5414.

Gröne, M., E. Hoffmann, S. Berchtold, B.R. Cullen, and R. Grassmann. 1994. A single stem-loop structure within the HTLV-1 Rex response element is sufficient to mediate Rex activity in vivo. Virology 204: 144-152.

Grüter, P., C. Tabernero, C. von Kobbe, C. Schmitt, C., Saavedra, A. Bachi, M. Wilm, B.K. Felber, and E. Izaurralde. 1998. TAP, the human homolog of Mex67p, mediates CTE-depen- 
dent RNA export from the nucleus. Mol. Cell 1: 649-659.

Hammarskjöld, M.-L. 1997. Regulation of retroviral RNA export. Cell Dev. Biol. 8: 83-90.

Herold, A., R. Truant, H. Wiegand, and B.R. Cullen. 1998. Determination of the functional domain organization of the importin $\alpha$ nuclear import factor. J. Cell Biol. 143: 309-318.

Hope, T.J., X. Huang, D. McDonald, and T.G. Parslow. 1990. Steroid-receptor fusion of the human immunodeficiency virus type 1 Rev transactivator: Mapping cryptic functions of the arginine-rich motif. Proc. Natl. Acad. Sci. 87: 77877791.

Hua, J., W. Blair, R. Truant, and B.R. Cullen. 1997. Identification of regions in HIV-1 Nef required for efficient downregulation of cell surface CD4. Virology 231: 231-238.

Huang, X., T.J. Hope, B.L. Bond, D. McDonald, K. Grahl, and T.G. Parslow. 1991. Minimal Rev-response element for type 1 human immunodeficiency virus. J. Virol. 65: 2131-2134.

Izaurralde, E. and S. Adam. 1998. Transport of macromolecules between the nucleus and the cytoplasm. RNA 4: 351-364.

Izaurralde, E., A. Jarmolowski, C. Beisel, I.W. Mattaj, G. Dreyfuss, and U. Fischer. 1997. A role for the M9 transport signal of hnRNP A1 in mRNA nuclear export. J. Cell Biol. 137: 2735.

Kadowaki, T., M. Hitomi, S. Chen, and A.M. Tartakoff. 1994. Nuclear mRNA accumulation causes nucleolar fragmentation in yeast mtr2 mutant. Mol. Biol. Cell 5: 1253-1263.

Lee, M.S., M. Henry, and P.A. Silver. 1996. A protein that shuttles between the nucleus and the cytoplasm is an important mediator of RNA export. Genes \& Dev. 10: 12331246.

Legrain, P. and M. Rosbash. 1989. Some cis- and trans-acting mutants for splicing target pre-mRNA to the cytoplasm. Cell 57: 573-583.

Malim, M.H., and B.R. Cullen. 1991. HIV-1 structural gene expression requires the binding of multiple Rev monomers to the viral RRE: Implications for HIV-1 latency. Cell 65: 241248.

Malim, M.H., J. Hauber, S.-Y. Le, J.V. Maizel, and B.R. Cullen. 1989. The HIV-1 rev trans-activator acts through a structured target sequence to activate nuclear export of unspliced viral mRNA. Nature 338: 254-257.

Malim, M.H., L.S. Tiley, D.F. McCarn, J.R. Rusche, J. Hauber, and B.R. Cullen. 1990. HIV-1 structural gene expression requires binding of the Rev trans-activator to its RNA target sequence. Cell 60: 675-683.

Malim, M.H., D.F. McCarn, L.S. Tiley, and B.R. Cullen. 1991. Mutational definition of the human immunodeficiency virus type $1 \mathrm{Rev}$ activation domain. J. Virol. 65: 4248-4254.

Meyer, B.E. and M.H. Malim. 1994. The HIV-1 Rev trans-activator shuttles between the nucleus and the cytoplasm. Genes \& Dev. 8: 1538-1547.

Michael, W.M., M. Choi, and G. Dreyfuss. 1995. A nuclear export signal in hnRNP A1: A signal-mediated, temperaturedependent nuclear protein export pathway. Cell 83: 415422.

Michael, W.M., P.S. Eder, and G. Dreyfuss. 1997. The K nuclear shuttling domain: a novel signal for nuclear import and nuclear export in the hnRNP K protein. EMBO J. 16: $3587-$ 3598.

Neville, M., F. Stutz, L. Lee, L.I. Davis, and M. Rosbash. 1997. The importin-beta family member Crm lp bridges the interaction between Rev and the nuclear pore complex during nuclear export. Curr. Biol. 7: 767-775.

Otero, G.C., M.E. Harris, J.E. Donello, and T.J. Hope. 1998. Leptomycin B inhibits equine infectious anemia virus Rev and feline immunodeficiency virus Rev function but not the function of the hepatitis B virus posttranscriptional regulatory element. J. Virol. 72: 7593-7597.

Pasquinelli, A.E., R.K. Ernst, E. Lund, C. Grimm, M.L. Zapp, D. Rekosh, M.-L. Hammarskjöld, and J.E. Dahlberg. 1997. The constitutive transport element (CTE) of mason-pfizer monkey virus (MPMV) accesses a cellular mRNA export pathway. EMBO J. 16: 7500-7510.

Piñol-Roma, S. and G. Dreyfuss. 1992. Shuttling of pre-mRNA binding proteins between nucleus and cytoplasm. Nature 355: 730-732.

Saavedra, C., B. Felber, and E. Izaurralde. 1997. The simian retrovirus-1 constitutive transport element, unlike the HIV-1 RRE, utilises factors required for the export of cellular mRNAs. Curr. Biol. 7: 619-628.

Santos-Rosa, H., H. Moreno, G. Simos, A. Segref, B. Fahrenkrog, N. Panté, and E. Hurt. 1998. Nuclear mRNA export requires complex formation between Mex67p and Mtr2p at the nuclear pores. Mol. Cell. Biol. 18: 6826-6838.

Segref, A., K. Sharma, V. Doye, A. Hellwig, J. Huber, R. Lührmann, and E. Hurt. 1997. Mex67p, a novel factor for nuclear mRNA export, binds to both poly $(\mathrm{A})^{+}$RNA and nuclear pores. $E M B O$ J. 16: 3256-3271.

Selby, M.J. and B.M. Peterlin. 1990. Trans-activation by HIV-1 Tat via a heterologous RNA binding protein. Cell 62: 769776.

Stade, K., C.S. Ford, C. Guthrie, and K. Weis. 1997. Exportin 1 $(\mathrm{Crm} 1 \mathrm{p})$ is an essential nuclear export factor. Cell 90: 10411050.

Stutz, F. and M. Rosbash. 1998. Nuclear RNA export. Genes \& Dev. 12: 3303-3319.

Tabernero, C., A.S. Zolotukhin, Z. Valentin, G.N. Pavlakis, and B.K. Felber. 1996. The posttranscriptional control element of the simian retrovirus type 1 forms an extensive RNA secondary structure necessary for its function. I. Virol. 70: 5998-6011.

Tang, H., G.M. Gaietta, W.H. Fischer, M.H. Ellisman, and F. Wong-Staal. 1997. A cellular cofactor for the constitutive transport element of type D retrovirus. Science 276: 14121415.

Tiley, L.S., S.J. Madore, M.H. Malim, and B.R. Cullen. 1992. The VP16 transcription activation domain is functional when targeted to a promoter-proximal RNA sequence. Genes \& Dev. 6: 2077-2087.

Truant, R., R.A. Fridell, E.R. Benson, A. Herold, and B.R. Cullen. 1998. Nucleocytoplasmic shuttling by protein nuclear import factors. Eur. J. Cell Biol. 77: 269-275.

Wen, W., J.L. Meinkoth, R.Y. Tsien, and S.S. Taylor. 1995. Identification of a signal for rapid export of proteins from the nucleus. Cell 82: 463-473.

Yoon, D., H. Lee, W. Seol, M. Demaria, M. Rosenzweig, and J.U. Jung. 1997. Tap: A novel cellular protein that interacts with Tip of Herpes virus Saimiri and induces lymphocytes aggregation. Immunity 6: 571-582.

Zolotukhin, A.S. and B.K. Felber. 1999. Nucleoporins Nup98 and Nup214 participate in nuclear export of human immunodeficiency virus type 1 Rev. J. Virol. 73: 120-127.

Zolotukhin, A.S., A. Valentin, G.N. Pavlakis, and B.K. Felber. 1994. Continuous Propagation of RRE(-) and Rev(-)RRE(-) human immunodeficiency virus type 1 molecular clones containing a cis-acting element of simian retrovirus type 1 in human peripheral blood lymphocytes. J. Virol. 68: 79447952. 


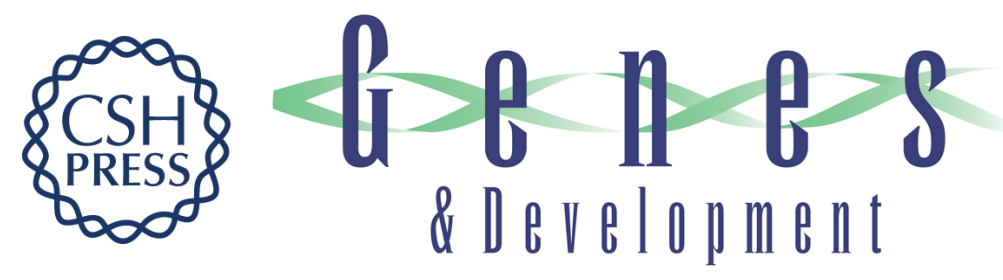

\section{The human Tap protein is a nuclear mRNA export factor that contains novel RNA-binding and nucleocytoplasmic transport sequences}

Yibin Kang and Bryan R. Cullen

Genes Dev. 1999, 13:

References This article cites 49 articles, 28 of which can be accessed free at:

http://genesdev.cshlp.org/content/13/9/1126.full.html\#ref-list-1

License

Email Alerting Receive free email alerts when new articles cite this article - sign up in the box at the top Service right corner of the article or click here.

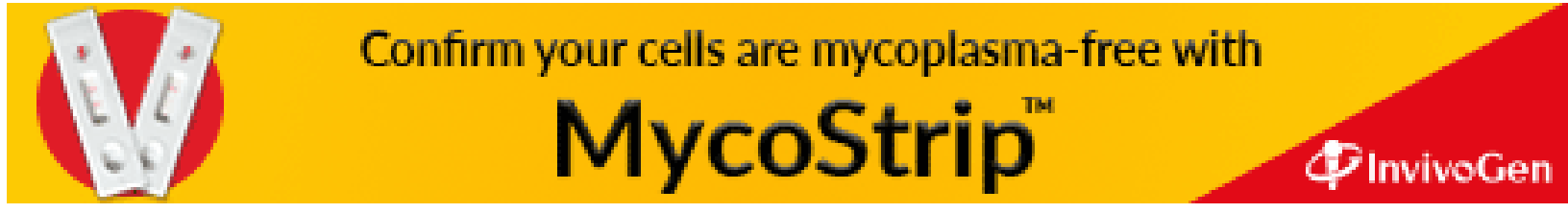

